\title{
The hybrid Brain-Computer Interface: a bridge to assistive technology?
}

\author{
Müller-Putz G.R. ${ }^{1,}$, Schreuder M. ${ }^{2}$, Tangermann M. ${ }^{2}$, Leeb R. ${ }^{3}$, Millán del R. J. ${ }^{3}$ \\ ${ }^{1}$ Institute for Knowledge Discovery, Graz University of Technology, Austria \\ ${ }^{2}$ Machine Learning Lab, Technische Universität Berlin, Germany \\ ${ }^{3}$ Chair in Non-Invasive Brain-Machine Interface, École Polytechnique Fédérale de Lausanne, Switzerland
}

gernot.mueller@tugraz.at

\begin{abstract}
Brain-Computer Interfaces (BCIs) can be extended by other input signals to form a so-called hybrid BCI (hBCI). Such an hBCI allows the processing of several input signals with at least one brain signal for control purposes, i.e. communication and environmental control.

This work shows the principle, technology and application of $h B C I s$ and discusses future objectives.
\end{abstract}

Keywords: hybrid BCI, electroencephalogram (EEG), assistive technology

\section{Introduction}

Persons with movement disabilities can use a wide range of assistive devices (ADs). The set of ADs ranges from switches connected to a remote controller to complex sensors (e.g., mouth mouse, eye tracking systems) attached to a computer. All of these systems work very well after being adjusted individually for each person. However, there are still situations where the systems do not work properly, e.g., when residual muscles become fatigued or users have such severe disabilities that no movement is possible. In such situations, a Brain-Computer Interface (BCI) might be the only available option, since it uses mentally modulated brain signals (e.g., electroencephalogram, EEG) for control without requiring any movement.

BCIs are systems that establish a direct connection between the human brain and a computer, thus providing an additional means for communication. As noted, some people use a BCI because their disabilities make it impossible to use any interface which requires movements. BCIs can also be used to control neuroprostheses in patients suffering from a high spinal cord injury. After 20 years of research and development, Brain-Computer Interface technology is ready to leave the lab and to be used in practical applications in real world settings such as homes or hospitals.

A BCI could replace an existing AD. However, in some situations it would be even better to couple the BCI with the existing $\mathrm{AD}$ and develop a new system called a hybrid BCI (hBCI) [1,2,8]. Ideally, an hBCI should let the user extend the types of inputs available to an assistive technology, or choose not to use the BCI at all. The hBCI might decide which input channel(s) offer(s) the most reliable signal(s) and switch between input channels to improve information transfer rate and usability, or it could instead fuse various input channels to increase the SNR.
In the past as well as in the present, various studies about hBCIs have been conducted, but they always combined different brain signals or brain and other biosignals. A more general definition says that a hBCI does not depend on the BCI as an active input. Instead, it simply allows the $\mathrm{BCI}$ to function as an input channel when the BCI could increase the overall performance for that user. The hBCI can perform fusion to switch between multiple inputs, but (depending on the configuration) can also weight signals and combine/fuse them to achieve one control signal from a combination of multiple inputs.

\section{Methods}

The principle of an hBCI can be explained as following: in addition to the EEG-based BCI, there are other input and control signals possible [2]. These include other biosignals (e.g., electromyogram, [3]) as well as signals from manual controls such as from ADs (e.g., mouth mouse, push buttons, ...[4,5]). A "fusion" collects all control signals and generates a new control signal out of all those inputs. Besides a quality check (e.g., artifact detection), those signals will be weighted and fused to a control signal, or the most reliable one will be chosen. Followed by the so-called "shared control", sensor signals from the application (neuroprosthesis [6], software [4], assistive robot [7]) will also be included and used to generate an accurate final control signal.

\section{Results \& Discussion}

One major goal is to bring the BCI technology to a level where it can be used in an environment together with other assistive devices. To achieve this, however, the hBCI must be able to operate reliably for long periods. It has to recognize and adapt to changes since brain signals and patterns may change during a day. Reaching this goal requires that many different subsystems in the $\mathrm{hBCI}$ are able to work together. Examples include standard BCI processing, post processing (error potentials), mental state recognition (fatigue), artifact detection, adaptation of classifiers, and surveillance of signal quality (including EEG signals and those from additional input devices). Several of those modules are developed and tested successfully, however, the integration of all of them is still an open issue. 


\section{Acknowledgement}

This work was supported by the EU FP7 projects TOBI (224631), and BackHome (288566) .

\section{Bibliography}

[1] Pfurtscheller, G.; Allison, B. Z.; Brunner, C.; Bauernfeind, G.; Solis Escalante, T.; Scherer, R.; Zander, T.; Müller-Putz, G.; Neuper, C.; Birbaumer, N.: The Hybrid BCI. - in: Frontiers in neuroprosthetics 4 (2010) , S. 1 11

[2] Müller-Putz, G.; Breitwieser, C.; Cincotti, F.; Leeb, R.; Schreuder, M.; Leotta, F.; Tavella, M.; Bianchi, L.; Kreilinger, A.; Ramsay, A.; Rohm, M.; Sagebaum, M.; Tonin, L.; Neuper, C.; del. R. Millán, J.: Tools for braincomputer interaction: a general concept for a hybrid BCI. - in: Frontiers in neuroinformatics 5 (2011) 30 , S. $1-10$

[3] Leeb, R.; Sagha, H.; Chavarriaga, R. \& d. R. Millán, J. A hybrid brain-computer interface based on the fusion of electroencephalographic and electromyographic activities. J Neural Eng, 2011, 8, 025011

[4] Clauzel, G.; Kaltner, B.; Breitwieser, C.; MüllerPutz, G.: Combining Hybrid BCI and signal quality monitoring to improve user experience. - in: 1st International DECODER Workshop. BoulogneBillancourt, Frankreich am: 11.04.2012

[5] Kreilinger, A.; Kaiser, V.; Breitwieser, C.; Williamson, J.; Neuper, C.; Müller-Putz, G.: Switching between manual control and brain-computer interface using long term and short term quality measures. - in: Frontiers in neuroscience 5 (2012) 00147 , S. 1 - 11

[6] Rohm, M.; Schneiders, M.; Kreilinger, A.; MüllerPutz, G.; Rupp, R.: First evaluation results of a BCIcontrolled hybrid neuroprosthesis for restoration of grasping in a high spinal cord injured individual. - in: Proceedings of TOBI Workshop lII. (2012), S. 8 - 9

[7] Tonin, L.; Carlson, T.; Leeb, R. \& d. R. Millán, J. Brain-Controlled Telepresence Robot by MotorDisabled People Proc. Annual International Conference of the IEEE Engineering in Medicine and Biology Society EMBC 2011, 2011, 4227-4230.

[8] Millán, J. d. R.; Rupp, R.; Müller-Putz, G.; MurraySmith, R.; Giugliemma, C.; Tangermann, M.; Vidaurre, C.; Cincotti, F.; Kübler, A.; Leeb, R.; Neuper, C.; Müller, K. \& Mattia, D. Combining Brain-Computer Interfaces and Assistive Technologies: State-of-the-Art and Challenges Frontiers in Neuroscience, 2010, 4, 161. 